\title{
MODEL STARS*
}

\section{BY HENRY NORRIS RUSSELL}

The distinguished record of the Willard Gibbs Lecture is more than enough to place a newcomer upon his mettle. In accepting the kind invitation of your President to add my contribution to the list, I must warn my hearers, as I warned him, that you will not hear today such a masterly discussion of major contributions made by your speaker as you have heard when the topic was "Relativity and Thermodynamics", or "Resonance in the Solar System." My humbler task must be to report upon the work of others in a field which I have only lightly touched.

The field itself is important enough, and the advances of knowledge in the past twenty years are sufficiently impressive. At that time we knew little, and dared not venture to guess much more, concerning the internal constitution of the stars; and some of the guesses which then looked best were wrong. Now we know enough to have interpreted some of the facts which then appeared hopelessly puzzling, and we still know little enough to afford room for very lively and enlightening controversy. Astronomy, physics, and mathematics have contributed to the advances. The astronomers contributed the observational data, and had already provided a great part of these, two decades since. Modern atomic theory laid the foundations for the new structure, while mathematical analysis - of a kind simple enough to the mathematicians, but little used previously in astronomy,--built it up.

So far has the work already progressed that it would far exceed the limits of this lecture, or of the time available for your lecturer's preparation--not to speak of those of your patience--. to give a detailed critique of the present situation. I will attempt only to give a general account of the problem, and deal in a sketchy fashion with some aspects of the solutions so far attempted, which elucidate something regarding the inner nature

* The Thirteenth Josiah Willard (iibbs Lecture, delivered at New York City, October 31, 1936, under the auspices of the American Mathematical Society, at a joint meeting of the Society and the American Physical Society. 
of the stars and something, too, regarding that of the investigators.

Perhaps the greatest problem of deductive physics may be posed as follows. Given a large quantity of matter, isolated in space, with all the necessary initial data regarding it, to find its subsequent history. Exhaustively handled, this would be a problem indeed. Its complete solution, according to the thorough-going mechanist, would render quite superfluous the whirl of straw-votes and political prognostications in which at the moment we are submerged. Leaving these high matters-or shall I say low?-and returning to the realms of dynamics and physics, let us note that the problem has been inadequately, and too comprehensively, stated. If the quantity of matter is supposed to be collected into a number of separate moving pieces, the study of their gravitational motions alone involves the whole problem of $n$ bodies, or, if we choose to carry it back into the past, the more difficult questions of cosmogony. Let us assume then, that our matter is concentrated into a single lump; and again that this lump is so big that the molecular forces between its least parts are insignificant in effect, compared with the gravitational forces which act upon it as a whole. This reduces the problem of equilibrium substantially to one of hydrostatics, and greatly simplifies the discussion.

We have now come to the astronomical problem; but it is still so big that it must be attacked piecemeal. The mass may be in rotation, whether like a rigid body or with angular velocity varying from part to part; or, even if it has no net angular momentum, it may not be initially in equilibrium, or at rest, and may subsequently undergo oscillations, complicated, in general, by the compressibility of the material. Consideration of these possibilities,- - which appear to be realized among actual, observable stars, and are of great astrophysical importanceshould evidently be deferred till the simpler case of the mass at rest has been discussed.

We have now a tractable problem of hydrostatics, since we have already assumed rigid forces to be negligible. The equilibrium configuration will obviously be spherically symmetrical, with concentric strata of equal density and the hydrostatic pressure $p$ will be given by the familiar equation

$$
d p=-\rho g d r \text {. }
$$


From the elementary relation an important consequence follows. We have in general, if $d v$ is the volume element,

$$
\int p d v=p v-\int v d p .
$$

Integrating from the center to the outer radius $R$, since the first term vanishes, we have

$$
\int p d v=\int_{0}^{R} \frac{4}{3} \pi r^{3} \cdot \rho \frac{G m}{r^{2}} d r=\frac{1}{3} \int_{0}^{M} \frac{G m d m}{r}=\frac{1}{3} \Omega,
$$

where $m$ is the mass inside a sphere of radius $r, M$ the whole mass, and $\Omega$ its potential upon itself.

Let us now assume that the pressure arises kinetically from molecular motions. Then $p$ is two-thirds the energy-density arising from the translational motions of the molecules, and if $E$ is the whole amount of such energy within the body, $E=\Omega / 2$. Hence for any mass maintained at rest in hydrostatic equilibrium by molecular motion, one-half the energy necessary to expand it to infinity against its gravitation is present in the translational motions of the molecules. The sum total of all the remaining available energy of the matter-of molecular rotation, chemical and ionization processes, and so on-must be less than the translational energy; otherwise the mass could liberate energy by diffusing altogether into space. For certain stars, as we shall see later, the margin of safety is small.

Further progress with our problem depends on the adoption of some relation connecting pressure and density. The simplest case- though by no means the first one to be discussed - is that of the cold body, a mass which contains no internal energy capable of transfer to another body, or radiation into space, in the form of heat. This is far from saying that it has no internal energy at all; the zero-point energy of the quantum theory remains.

For low pressures, when cold matter is crystalline, the expression for this is complicated, but at great pressures and high densities it becomes simple, for the matter is no longer crystalline, but degenerate.

I believe that Bridgman was the first to point out that a sufficiently great hydrostatic pressure, at however low a tem- 
perature, should suffice to break down the quantized structure of "electron orbits" in the outer parts of atoms, and reduce the whole mass to a swarm of separate electrons and nuclei. The quantum relations are still active, and the same restriction which ordinarily limits the number of electrons in a given shell now demands that not more than so many slow-moving electrons may be crowded into a given volume. When this state of degeneracy has been fully reached, the pressure and density are connected by the relation*

$$
p=K_{1} \rho^{5 / 3}
$$

where

$$
K_{1}=\frac{1}{20}\left(\frac{3}{\pi}\right)^{2 / 3} \frac{h^{2}}{m\left(\mu^{\prime} H\right)^{5 / 3}}=9.86 \times 10^{12} \mu^{\prime-5 / 3}
$$

in c.g.s. units, $H$ is the mass of the unit of atomic weight, $m$ that of the electron, and $\mu^{\prime}$ is the mean mass, in atomic-weight units, per free electron in the completely ionized material. Here only the electrons are supposed to be degenerate. Degeneracy for the nuclei, though possible, does not come within the range of our discussion.

The equations (1) and (3) suffice to determine the configuration of the mass completely. They present a particular case of the polytropic equilibrium which was thoroughly discussed by Emden, $\dagger$ in a memoir which has had great influence.

If, in general, we set

$$
p=K \rho^{1+1 / n},
$$

and if we set $\theta^{n}=\rho, \xi=r[(n+1) / 4 \pi G]^{1 / 2}$, we find from (1),

$$
\frac{d^{2} \theta}{d \xi^{2}}+\frac{2}{\xi} \frac{d \theta}{d \xi}+\theta^{n}=0 \text {. }
$$

This is Emden's equation. For $n=0$ and $n=1$ it is easily integrated. For other values of $n$ it defines new transcendental functions, though for $n=5$ it admits of the solution $\theta=\left(1+\xi^{2} / 3\right)^{-1 / 2}$. The solutions of this equation have been extensively studied,

* E. A. Milne, Monthly Notices of the Royal Astronomical Society, vol. 91 (1930), p. 30.

$\dagger$ Gaskugeln, Leipzig, 1907. 
especially by R. H. Fowler.* For a given value of $n$ there is a double infinity of solutions. For the vast majority of these $\theta$, when carried inward towards $\xi=0$, either increases without limit, or passes from originally positive values to negative. Only for a simple infinity of solutions is $\theta$ finite when $\xi=0$ (and $d \theta / d \xi=0)$. All these solutions may be derived from the one for which $\theta=1, \xi=0$ by multiplying $\theta$ and dividing $\xi$ by appropriate scale-constants. These are obviously the only solutions which give results of physical significance, when applied to the whole of a spherical mass, though other solutions may apply to the outer parts of a sphere containing a core defined in some other way. With this limitation each value of the polytropic index $n$ defines a perfectly definite law of distribution of internal density within the mass-which we may describe as the model on which it is built.

These solutions were accurately computed by quadratures, by Emden, and his calculations have been extended, with still greater precision, under the direction of Comrie. The value $n=0$ gives a homogeneous sphere. As $n$ increases, there is a steadily growing concentration of density toward the center, the ratio of the central to the mean density being as follows:

\begin{tabular}{ccccccccccc}
\multicolumn{2}{c}{$n=0$} & 1 & 1.5 & 2 & 2.5 & 3 & 4 & 4.5 & 4.9 & 5 \\
$\rho_{c} / \rho_{m}$ & 1.00 & 3.29 & 6.00 & 11.40 & 24.08 & 54.3 & 623 & 6378 & $9.35 \times 10^{5}$ & $\infty$
\end{tabular}

For $n=5$ the sphere extends to infinity, and for greater values of $n$ there is no finite solution with finite central density.

These polytropic solutions have had a remarkable influence on later work in this field. In certain cases they appear naturally as a result of definite physical assumptions; for example, the "ordinary" degeneracy of equation (3) gives $n=3 / 2$, and Eddington's famous solution $n=3$. There is no evidence, however, that they are in general inherently adapted to the solution of problems of the present sort, as the ordinary trigonometric functions, spherical harmonics, Bessel functions, and so on, are in well known cases; and there can be little doubt that, in many instances, the convenience of having a set of functions already computed has led theoretical workers to design their postulates so that they would be led to polytropic functions rather than to the very heavy labor of new quadratures.

\footnotetext{
* Monthly Notices of the Royal Astronomical Society, vol. 91 (1930), p. 63.
} 
In any case, the polytropic functions are well suited for choice as a generalized set of density-models, since, with a moderate range of the parameter $n$, they represent all degrees of central condensation. It may finally be noted that at the boundary of such a sphere, $\theta, \rho$, and $p$ vanish, while $d \theta / d \xi$ remains finite, though approaching zero as $n$ approaches 5 .

Returning now to the degenerate cold body, we note that its central density is six times the mean density - not a high degree of concentration. If the mass $M$ and molecular weight $\mu$ are given, all the properties of the body are completely determined, as was first pointed out by Milne.

In terms of the sun's mass and radius, it is found that

$$
\frac{R}{R \odot}=0.040\left(\frac{M}{M_{0}}\right)^{-1 / 3} \mu^{\prime-5 / 3} .
$$

For masses comparable to the sun's, the mean density is of the order of $15000 \mu^{5}$.

The white dwarf stars have densities of this order, which is just what might be expected if they are approaching, but have not yet reached, the loss of their internal heat. It is practically certain that they have highly degenerate cores, shading off into normal gas at the surface. Twenty years ago these stars presented the most intractable - indeed, apparently unintelligible-problem in astrophysics. Today they are closer to a complete theoretical solution than any others.

When the pressure and energy-density become extremely great, and the velocities of the electrons approach that of light, a new stage of relativistic degeneracy gradually sets in. When it is complete, the equation of state is*

$$
p=K_{2} \rho^{4 / 3}, \quad K_{2}=\left(\frac{3}{\pi}\right)^{1 / 3} \frac{h c}{8\left(\mu^{\prime} H\right)^{4 / 3}}=1.23 \times 10^{14} \mu^{\prime-4 / 3} .
$$

This leads to a polytrope $n=3$, with central density 54 times the mean. In Eddington's opinion, the arguments which lead to this equation of state are erroneous, and equation (3) should hold good for all densities. Practically all other investigators

* Chandrasekhar, Monthly Notices of the Royal Astronomical Society vol. 91 (1931), p. 458. 
disagree with this conclusion, and the general consensus will here be followed.

The transition between the two types of degeneracy has been worked out in detail by Stoner* and Chandrasekhar, who give the exact equations

$$
\begin{aligned}
P & =\frac{\pi m^{4} C^{5}}{3 h^{3}}\left[x\left(2 x^{2}-3\right)\left(x^{2}+1\right)^{1 / 2}+3 \sinh ^{-1} x\right] \\
\rho & =\frac{8 \pi m^{3} c^{3} \mu^{\prime} H}{3 h^{3}} x^{3} .
\end{aligned}
$$

Chandrasekhar, by an elegant piece of analysis, $\dagger$ has shown that in this case Emden's equation must be replaced by

$$
\frac{1}{\eta} \frac{d}{d \eta}\left(\eta^{2} \frac{d \phi}{d \eta}\right)=-\left(\phi^{2}-\frac{1}{y_{0}^{2}}\right)^{3 / 2}
$$

where $y^{2}=x^{2}+1, r=\alpha \eta, y=y_{0} \phi$, and $\alpha$ and $y_{0}$ are constants. This equation takes exact account of the onset of relativistic degeneracy. Chandrasekhar has integrated it by quadratures. The radius depends on the mass in the following fashion (taking the sun's mass and radius as units).

$\begin{array}{lrrrrrrrr}M^{2} \mu^{\prime 2} & 0.88 & 1.61 & 2.44 & 3.53 & 4.31 & 5.29 & 5.48 & 5.73 \\ r \mu^{\prime} & 0.040 & 0.031 & 0.025 & 0.019 & 0.014 & 0.008 & 0.006 & 0.000\end{array}$

A new phenomenon here appears-the limiting mass $M_{0}$ $=5.73 / \mu^{\prime 2}$. For smaller masses the limiting radius, though small, is definite. For $M=M_{0}$, it is zero; while for larger masses, as, Chandrasekhar shows, complete degeneracy cannot occur, since, even with the limiting model $n=3$, the energy-density required to balance the gravitational pressure exceeds that of the zeropoint state, so that the mass can never be internally cold. The ratio of the two is independent of the radius, and no limit exists.

Incidentally, in the relativistically degenerate state the pressure is one-third of the energy density, so that the whole of the energy of gravitational contraction will be exhausted in keeping up the pressure. A body of mass $M_{0}$, once it became relativisti651.

* Monthly Notices of the Royal Astronomical Society, vol. 92 (1932), p.

$\dagger$ Ibid., vol. 95 (1935), p. 207. 
cally degenerate, would therefore contract rapidly, with no need of getting rid of any surplus energy by radiation, while an ordinarily degenerate mass would have to radiate away half of the gravitational supply - which, in view of the low luminosity of white dwarfs, would take an enormously long time.

The final state of a larger mass can only be conjectured. It is, of course, physically absurd to suppose that its density would become infinite. Whether, and when, the electrons or nuclei would be jammed so closely that their own effective volumes would have to be considered, cannot be definitely stated. Something would have to happen, anyhow, by the time that the radius of the body was reduced to the relativisitic equivalent of its mass (about one mile for the sun)-but some one else had better say what would happen!

For small masses, the formula based on degeneracy must also fail. A ton of matter, for example, cooled toward the absolute zero, would undoubtedly settle down as a crystalline mass of density not much different from that at higher temperatures, and the same should be true of bodies the size of asteroids, or even of planets, though, in the latter case, the gravitational pressure would considerably increase the mean density.

For such bodies, the radius obviously increases with the mass. In the degenerate case, the radius decreases with increasing mass. There must therefore be some intermediate mass for which the radius of a cold body is a maximum, and no body in the universe can be larger than this, unless it is hot inside. Kothari and Majumdar, ${ }^{*}$ following a suggestion of the speaker, conclude that the critical mass is about $1 / 200$ the sun's and the radius about $20,000 \mathrm{~km}$. The problem of the cold body is determinate when once the total mass and the chemical composition are given, provided that the mass is great enough to produce ionization by pressure, and degeneracy, throughout most of its extent. Variations in composition will then affect only the mean molecular weight $\mu$. For small masses, in which ordinary compounds occur, the complexity may be great; but such masses are observable only within the solar system.

For hot bodies, the equation of state contains a new parameter- the temperature-, and the increased freedom corresponds to the introduction of an arbitrary function into our

* Nature, vol. 137 (1936), p. 157. 
solutions. Suppose, for example, that we are dealing with a perfect gas for which $p=R \rho T / \mu$, where $R$ is the gas constant, and $\mu$ the mean molecular weight per free particle (including electrons, ions, neutral atoms, and molecules). We may represent any arbitrary spherical distribution of density by the equation

$$
\rho=\rho_{0} X,
$$

where $\rho_{0}$ is the mean density $(3 / 4 \pi) M r^{-3}$, and $X$ is a function of $r$, arbitrary except for a normalization factor. The hydrostatic pressure $P$ at any point follows by quadratures, and may be expressed in the form

$$
P=P_{0} Y
$$

where

$$
P_{0}=\frac{3 G}{8 \pi} M^{2} r^{-4}
$$

and is the pressure at the center of a homogeneous sphere, and $Y$ is a function of $r$, completely determined when $X$ is given. The gas-equation then gives

$$
T=\frac{\mu G}{2 R} \frac{Y}{X} \frac{M}{r} .
$$

If the temperature at any point has this value, a sphere of any arbitrary density-model $X$ will be in hydrostatic equilibrium throughout. With the numerical values for the sun,

$$
T=1.15 \times 10^{7} \frac{\mu Y}{X} \text { (in Centigrade degrees) } .
$$

We have therefore to deal throughout practically the whole interior of the sun (and of the stars at large), with temperatures enormously too high to permit the formation of compounds, with the great resulting complications. Indeed, the atoms themselves will be very highly ionized - stripped almost to their nuclei. Under these conditions, the mean molecular weight $\mu$ is nearly the same for most of the elements, ranging from 1.9 for carbon to 2.3 for iron (as the calculations of Fowler and Guggenheim show in detail). For hydrogen, however, $\mu=0.5$.

This greatly simplifies the discussion. The chemical com- 
position, if we include all known atomic species, is a function of 90 variables. In practice this number could be reduced to about a dozen by ignoring rare elements, and grouping others with similar properties. But for the present purpose atoms fall into two classes, hydrogen and the others, and the specification of the abundance of hydrogen suffices for a first approximation.

A second, and still more important, consequence of the high temperature is that radiation pressure becomes significant (as Eddington was the first to realize). The hydrostatic pressure of radiation in an enclosure of temperature $T$ is $q=a T^{4} / 3$, $\left(a=7.6 \times 10^{-15}\right)$. If we set $p=\beta P, q=(1-\beta) P$, it follows that

$$
\frac{1-\beta}{\beta^{4}}=\frac{\pi}{18} \frac{a G^{3}}{R^{4}} \frac{Y^{3}}{X^{4}} \mu^{4} M^{2}=0.0325 \frac{Y^{3}}{X^{4}} \mu^{4}\left(\frac{M}{\odot}\right)^{2},
$$

where $\odot$ is the sun's mass. The internal constitution of a gaseous star built on any arbitrarily assigned density-model is now completely determined, the temperature at any point being $\beta$ times the value given by (10). An exact solution must take the variations of $\mu$ into account; but the ionization, and hence $\mu$, at any point depends only on the local temperature and pressure, and the composition of the material. If the latter is known, along with the pressure and density, the values of $\mu, \beta$, and $T$ are determinate, and may be found by trial and error.

In the absence of any knowledge of the composition, our exact solution still involves an unknown function of ninety variables! To avoid hopeless complications, it is customary to assume that the material within our body is well mixed and of uniform composition throughout. In this case the problem becomes quite definite; but whether the composition of the very thin surface layer which can be reached with the spectroscope is typical of the interior of a star must be settled, if at all, in other ways.

Though a mass of gas of uniform composition may be in equilibrium with any arbitrary density model, it need not be stable. Models in which the density decreases inward at any point, for example, are obviously unstable, for vertical convection currents would lead to a rearrangement of lower potential energy. The compressibility of the gas makes things worse, and the familiar condition for convective stability is that the temperature gradient shall be less than that value corresponding to an adiabatic transfer of gas from the higher to the lower pres- 
sure. When changes in the internal energy of the gas are occurring - for example, when its degree of ionization altersconvection may set in despite a considerable temperature gradient. Unsöld has shown, for example, that a convective region may occur just under the photosphere of the sun, where the hydrogen, highly ionized in depth, is almost entirely neutral at the surface, and thus the resulting currents, ascending above the top of the convective layer, and cooled by expansion in their last stages, give a reasonable explanation of sun-spots. Convection regions, with some models, may also occur in the deep interior.

So far, we have ignored one rather important property of our model stars: we have not bothered to ask whether and how brightly they will shine. They must shine, for they are hotter inside than outside, and heat will flow from the hotter to the cooler regions. The three familiar processes of transfer, conduction, convection, and radiation, must all be considered; but, under stellar conditions, the last is usually so much more effective than the others that they may be neglected.

If neutrons, with their long free paths, were abundant in the interior, conduction might be important, but consideration of this may be deferred until nuclear physicists have settled the question whether neutrons are stable enough to exist permanently in a medium where collisions are numerous and violent.

The equation of radiative transfer of energy may be written

$$
H=-\frac{c}{k \rho} \frac{d q}{d r},
$$

where $c$ is the velocity of light, and $k$ the mass-coefficient of opacity of the medium. In the deep interior of stars, this arises mainly from the photo-electric ionization of the partly stripped atoms by incident photons-which for the most part have the energy and wave-length of soft X-rays. Scattering by free electrons plays a subordinate part. An exact calculation of opacity would be difficult, demanding a study of the energy-levels of the individual atomic types, and an integration of the effect over all wave-lengths, weighted according to the distribution of the radiation; but a good approximation can be obtained by smoothing out the individual peculiarities. The results of Kramers and Gaunt, which are generally accepted, lead to the formula 


$$
k=C \rho T^{-7 / 2} .
$$

The coefficient $C$ depends on the composition of the gas. Hydrogen and helium, which are practically always reduced to bare nuclei, contribute very little directly to the opacity, though the electrons released from them do their share.

For the total flux of radiation outward through a sphere, inside the star, of radius $r$, we find

$$
l=-\frac{4 \pi c r^{2}}{k \rho} \frac{d q}{d r} .
$$

This may be calculated for any model, and gives the flow of heat corresponding to the assumed conditions. If there are no internal sources of heat, the temperature and other properties of the mass-including in general the law of distribution of densitywill change with the time. For a body which, like most stars, has a store of internal heat millions of times the annual radiation, the changes will be slow.

If our model is to represent a star, we must endow it with sources of heat sufficient to supply the flux. The rate of liberation of heat, per unit mass, within the shell of radii $r$ and $r+d r$ must be

$$
\epsilon=\frac{1}{4 \pi r^{2} \rho} \frac{d l}{d r} .
$$

For a model chosen at random, $\epsilon$ is likely to be negative in some regions, which must be supposed to contain sinks, rather than sources, of available energy. This assumption does not appear to be physically absurd, nor impossible; but it is aesthetically unattractive, and is very unpopular among model-makers. Until some specific reason can be given why, under specified conditions, heat or radiant energy should be frozen into some unavailable form, this prejudice may well be respected.

The exclusion of heat-sinks places considerable restrictions upon the permissible models-for example, it rules out the outer parts of many polytropic distributions-; but it still leaves our problem indeterminate by an arbitrary function.

The solution becomes definite if the law of generation of energy is known. We may take $p, q$ as independent variables, and have 


$$
\begin{array}{rlrl}
\rho & =\rho(p, q, \mu), & & \text { equation of state. } \\
k & =k(p, q, \mu), & & \text { equation of opacity. } \\
\epsilon & =\epsilon(p, q, \mu), & & \text { equation of heat generation. } \\
d m=4 \pi \rho r^{2} d r, & d p=\frac{k l-4 \pi c G m}{4 \pi c r^{2}} \rho d r, \\
d l=4 \pi \epsilon \rho r^{2} d r, & d q=-\frac{k l}{4 \pi c r^{2}} \rho d r .
\end{array}
$$

In the equations of state, and so on, $\mu$ has been introduced as a sort of shorthand for the composition of the material. It actually represents a most formidable complexity, for there is the best of reason to believe that different isotopes may behave differently as regards the liberation of nuclear energy, so that, in general, $\mu$ stands for a function of more than two hundred variables, whose relative influence may vary in an unknown fashion with $r$.

We may cut the Gordian knot by assuming as before that the material is well mixed and of constant atomic and isotopic composition. Then $\mu$ becomes a definite, though complicated, function of $p$ and $q$, depending on the ionization, and $\rho, K$, and $\epsilon$ are also known functions of $p, q$.

We may assume them to be one-valued and completely definite, with no disposable parameters. This would not be true for the equation of state if two or more phases were present (as for a liquid at its boiling point); but the high temperatures of the stars, even at their surfaces, dispel the fear of such complications.

The assumption that the opacity depends entirely upon the local values of $p$ and $q$ is not universally true, for $k$ is a harmonic Rosseland mean of the values for different frequencies. If the incident radiation came from regions at a considerable distance, as is the case in a planetary nebula, or the envelope of a nova, its spectral composition would depend on conditions in its source. Inside a star, however, the opacity is considerable in a distance of one meter, and the differences between the local circumstances and those in the region from which sensible direct radiation comes are entirely negligible. 
More questionable is the assumption that $\epsilon$ depends only on local conditions. It appears to be legitimate for the liberation of energy from sub-atomic sources-for cosmic rays, the only known external agents which might produce effects of this order, do not penetrate beyond the merest surface of a star. But it is certainly not true for the next largest known source of energy, gravitational contraction. The rate of exhaustion of potential energy is proportional-other things being equal-to the rate of contraction of the mass and is altered by any concomitant change in the density-model, while the rate of storage of energy by changes in ionization, and so on, depends on the rate of change of the local conditions. The whole analytical treatment, in which it has been tacitly assumed that the size of the mass and the internal distribution of matter do not change with time, is in this case inapplicable.

Even sub-atomic energy must ultimately be exhausted, and no star which shines can therefore be in a perfectly steady state. But the time-scale of the resulting changes is so long that the neglect of the undoubted diminution of mass, and the probable changes in composition, presumably introduces no significant errors.

We will now treat the functions $\rho, k, \epsilon$ as one-valued and completely definite, and devoid of poles or other singularities for finite values of $p, q$. This last assumption is necessary if they are to represent physically real quantities; the former will fail for sufficiently low values of $q$, that is, of the temperature. By excluding this range, we will limit the applicability of our results to actual stars very little, if at all. For very high temperatures and pressures the form of the functions is imperfectly known; but the assumption that their values are finite and definite ought still to be sound.

We have now a system of differential equations of the fourth order. For any given $r$, we may assign any desired initial values of $p, q, l, m$, and follow the corresponding solution indefinitely by analytical continuation, or by numerical quadratures, which is another name for the same thing. Our solution will have a physical meaning, so long as $p, q, l, m$ are all positive. Within these limits, it may be interpreted as representing the configuration of a mass of matter, limited internally by a rigid sphere of 
mass $m$, emitting the radiation $l_{1}$, and externally by a rigid spherical wall absorbing the radiation $l_{2}$ and sustaining a pressure $p_{2}+q_{2}$.

But if our model is to represent an autonomous star, it must satisfy certain boundary conditions. Two of these come at the center; we must have $l=0, m=0$, when $r=0$, since pointmasses and point-sources of energy have no physical sense.

The other applies at the outer boundary, and is less easy to describe with precision. It may be approximately stated by defining the boundary as the value of $r$ for which $p=0$, and assuming that $q=0$ at the same point. But the actual temperature at and above a star's surface is high. The equilibrium of the outer layer, which presents a simpler problem than that of the interior, has been fully studied and it is found that the temperature falls asymptotically toward a limit $T_{0}$ which is $(3 / 16)^{1 / 8}$ or 0.885 times the effective temperature $T_{b}$ defined by the flux of radiation escaping per unit area from the surface. The outer atmosphere of the star is nearly isothermal, and the decrease of density with height is approximately exponential.

For a high value of gravity, such as prevails on the sun and most stars, the density drops from a considerable value to practically zero in less than a thousandth of the radius, so that, for practical purposes, the star has a sharp boundary. If $q_{0}$ is the value of the radiation pressure corresponding to $T_{0}$, we may define our boundary condition by saying that $q$ approaches $q_{0}$ as $p$ approaches zero. This is not a rigorous statement, for the equation of energy-transfer requires some modification in the outer atmosphere, but it is amply sufficient in practice.

Indeed, the internal temperatures of the stars are so much greater than those at the surface that two solutions of the fundamental equations - one starting with $p=0, q=0$, and the other with $p=0, q=q_{0}$ - when carried inward would soon differ by less than a millionth part, so that the simpler form of the boundary condition is practically sufficient.

In a generalized study of autonomous gas-masses this would not be permissible. There may be solutions corresponding to bodies of such small density as to be translucent to a considerable fraction of the radius. For these the diminution of gravity in the upper parts of the atmosphere has to be considered; and 
the solution is much more complicated; but they are model nebulae, and not model stars.

We might also consider the possibility of an outer envelope supported by differential radiation pressure, such as is directly observable in the solar chromosphere, and has been detected photometrically and spectroscopically about the remarkable eclipsing variable Zeta Aurigae; but the pressure at the base of such an envelope is utterly negligible from our present standpoint.

Out of the four-fold infinity of solutions of our system of differential equations, only a single infinity will satisfy the three independent boundary conditions. The model stars which can be formed out of a precisely specified material therefore form a oneparameter family. For any given value of any property of such a model, for example, its mass, all its other characteristics-such as radius, luminosity, internal distribution of density-will be completely defined. There may of course be more than one solution for the given mass, but the solutions will be discrete and definite. If any two properties of the series of models, for example, mass and radius, luminosity and effective temperature, are plotted as coordinates, the resulting points will lie on a definite curve.

This important theorem is due to Vogt.* It has immediate practical applications. On the familiar diagram in which the absolute magnitudes of the stars are plotted against the spectral types, the majority of the points lie on a narrow band-the main sequence-extending from the hot and luminous B-stars to the cool, faint $M$ dwarfs. This sequence may represent a series of stars of similar composition but different masses. The scatter of the points about the median line is too large to attribute to observational error, but is just what might be expected from minor differences in composition. The other important sequence (the normal giants) may perhaps represent a series differing in composition-or dependent on some more easily liberated source of energy. But all across the upper part of the diagram are points representing the super-giant stars, which are scattered almost at random over a large area. These suggest very strongly that, among the stars of great mass and

\footnotetext{
* Astronomische Nachrichten, vol. 226 (1926), p. 301.
} 
luminosity, there are important differences of composition or of energy-generation involving at least one continuously varying parameter. It may be, as Strömgren and Kuiper have suggested, that the properties of stars of small mass are much less sensitive to this parameter, and that the narrow spread along the lower part of the main sequence corresponds to the wide dispersion of the super-giants; but this is still speculative.

I must come back from real stars to models. The actual computation of the set of models corresponding to given functions $\rho, k, \epsilon$, would be exceedingly laborious. One starts, of course, at $r=0$, with promising assumed values of $p$ and $q$, and determines $m$ and $l$, and then $d p / d r$ and $d q / d r$, by quadratures of the usual sort. Two of the three boundary conditions are thus included at the start. The integration is continued until $p$ (or $q$ ) approaches zero, or begins to run wild. It would be an extraordinary piece of luck if the initial values should be chosen so that $p$ and $q$ vanished simultaneously. If they do not, the quadratures are repeated, starting, for example, with the same value of $q$ and another of $p$; and this process of trial and error is continued until the boundary condition is bracketed and then reached by interpolation. This gives a model star with assigned central temperature. Repeating the whole process for various values of $q$, the series of models can ultimately be obtained.

It is not surprising that no complete series of such models has so far been calculated. A few interesting cases, based upon plausible laws of heat-generation, have been worked out by Steensholt, Biermann, and others, but not enough has yet been done to reveal fully the theoretical way in which the radius and surface temperature change with the mass.

The time is hardly ripe, indeed, for so great a task; for very little is yet known regarding the rate of liberation of sub-atomic energy which should occur in a gas of specified composition, pressure, and temperature.

It is now generally believed that the source of stellar energy is to be found in the transmutation of hydrogen, or of neutrons, into heavier atoms, with disappearance of the excess of mass possessed by the proton or neutron, and liberation of the corresponding amount of energy. The alternative hypothesis, the annihilation of atoms, with transformation of the whole mass into energy, is now little considered, since there is no evidence, 
theoretical or observational, that it may occur, while a host of reactions of the other sort have recently been produced in the laboratory, and studied in detail.

When the laws which govern these processes have been fully worked out, it may be possible to predict $\epsilon$ theoretically and proceed to a complete and rational theory of the stars; but enough is already known to indicate that a fairly detailed knowledge of the atomic and isotopic composition of the material will be a prerequisite for any precise calculation.

Empirical models of stellar interiors will probably remain useful for a good while to come, and we may well discuss some of these.

First in order of time, and, even yet, of importance, is Eddington's "standard model," a name given by his friendly critic, Milne. Let $L, M$ be the values for the whole star, and let $l / m=\eta L / M$. Then, by $(15)$, since $m=-\left(r^{2} / G \rho\right)(d P / d r)$,

$$
k \eta=\frac{4 \pi c G M}{L} \frac{d q}{d P} .
$$

When Eddington originally derived this relation in 1916, nothing definite could be theoretically predicted about either $k$ or $\eta$, and the assumption that each was separately constant had the merit of simplicity, and also of giving a directly integrable equation. If $k \eta=K_{0}$, we have

$$
q=(1-\beta) P, \quad 1-\beta=\frac{K_{0} L}{4 \pi c G M},
$$

where the constant of integration is taken to be zero, on account of the low surface temperature. Substituting in equation (12), we have

$$
\frac{Y^{3} \mu^{4}}{X^{4}}=\text { const } . \quad \text { or } \quad \frac{P^{3} \mu^{4}}{\rho^{4}}=\text { const. }
$$

If $\mu$ is constant throughout the mass, this leads to $P \propto \rho^{4 / 3}$, and to the Emden polytrope $n=3$, and the model is completely determined, the central density being 54 times the mean.

We may take account of variations of $\mu$ qualitatively by assuming that $\mu \propto \rho^{x}$, and get a polytrope of index $3 /(1-x)$. This 
is of course an ad hoc procedure to secure a polytrope, while the original assumptions led to one naturally; but its value appears when we note that the ionization decreases, and $\mu$ increases, toward the surface, which demands a negative $x$, a polytropic index less than 3 , and a smaller central condensation.

We now have $L=\left(4 \pi c G / K_{0}\right) M(1-\beta)$. For this polytrope $Y^{3} / X^{4}=0.091$, so that

$$
\frac{1-\beta}{\beta^{4}}=0.0031 \mu^{4}\left(\frac{M}{\odot}\right)^{2} .
$$

The last two equations express the renowned mass-luminosity relation. Determining $K_{0}$ empirically, from the accurate data for Capella, Eddington found that his curve represented the reliable data then existing for the whole range of masses, unexpectedly including the faint and dense red dwarfs, and was thus led to the realization that the high ionization inside the stars caused the material to behave practically like a perfect gas, even at very high density.

With the physical law $k \propto \rho T^{-7 / 2}$ and the polytrope $n=3$, the condition $k \eta=$ const. demands sinks of energy near the surface. This difficulty can be avoided by altering the model for the outer part of the star, with but small change in the massluminosity relation. In the main part of the interior, $\epsilon$ increases rather slowly toward the center, being roughly proportional to $T$.

If we keep $\eta$ constant, assume the general polytropic relation $T \propto \beta \theta, \rho \propto \theta, P \propto \theta^{n+1}$, and confine ourselves to stars of small masses, we find easily that $n=3 \frac{1}{4}$, a result due to Jeans. This polytropic solution, however, does not hold good for the whole model, for $(1-\beta) / \beta^{4} \propto \theta^{-1 / 4}$ and becomes so large near the surface that an approximation fails. Only the separate assumptions $k=$ const., $\eta=$ const. lead therefore to a strictly polytropic solution.

Another interesting model is that in which the whole liberation of energy is supposed to take place in a point-source at the center. The solution must be made by quadratures, and the first computations were made by Eddington. Some general properties were later worked out analytically by Cowling.* Near the

\footnotetext{
* Monthly Notices of the Royal Astronomical Society, vol. 91 (1930), p. 92.
} 
center of this model is a hole where the enormous radiation pressure drives the matter away, and leaves almost a vacuum. Though physically unreal, this model is useful as the limiting case of extreme concentration of the energy sources in the inner and hotter regions.

Eddington's calculations - made for a single case, with mass 5.02 times the sun's - revealed one important fact. The luminosity of the point-source model was fainter by only 0.97 stellar magnitudes than that of a standard model of the same mass, radius, and composition. For models of the latter type, with masses ranging from 0.2 to 20 times the sun's-about the actual range among the stars-the luminosity varies by 16 magnitudes. The effects of the great change in the model are almost negligible in comparison.

Later investigations have fully confirmed this conclusion. For stars of the same composition, the mass-luminosity relation is extraordinarily insensitive to variations in the model. The fullest discussion is Vogt's.* He finds the relation (in our notation)

where

$$
l=\frac{4 \pi c G}{k} m(1-\beta)\left[1+\frac{\beta}{4-3 \beta} \chi(r)+\frac{d \log \mu^{4}}{d \log P}\right],
$$

$$
\chi(r)=\frac{d \log \left(\frac{Y^{3}}{X^{4}}\right)}{d \log P}=3-4 \frac{d \log X}{d \log Y} .
$$

For homologous stars, with the same density-model, $\chi(r)$ is the same at homologous points. If the values of $\beta, \chi, \cdots$ are taken for a sphere enclosing, say, 99 per cent of the mass, the luminosity of the star as a whole will be reproduced within the errors of observation. The term $d \log \mu^{4} / d \log P$ is small. It is possible to design a model such that $\chi(r)$ has any value; but it will usually be small. For example, for the polytrope of index $n$, $\chi=(3-n) /(1+n)$, and changes only from +0.60 to -0.33 as $n$ runs from 1.5 to 5 , which includes the range of central condensation likely to be met with. The greatest corresponding change in stellar magnitude is $-0^{m} .95$, when $\beta=1$.

If the zero-point of the mass-luminosity relation is deter-

\footnotetext{
* Veröffentlichungen der Universität-Sternwarte zu Jena, vol. 3 (1930).
} 
mined from the stars themselves, the course of the curve will be almost the same for a great variety of models. The conspicuous agreement of observation with this curve, which is fully confirmed by all the data now available, is therefore strong evidence in favor of Eddington's general theory of stellar luminosity, but not diagnostic in favor of his original model, or any other.

For a given model and mass, the predicted luminosity is greatly influenced by the assumed mean atomic weight $\mu$, since $1-\beta \propto \mu^{4} \beta^{4}$. For a mass composed predominantly of hydrogen, $\mu$ is close to 0.5 ; for one of heavy atoms, it is about 2.2 , and $\mu^{4}$ changes by a factor of 370 , corresponding to 6.4 magnitudes. With increasing percentage of hydrogen, the luminosity rapidly diminishes. For a mass of almost pure hydrogen, the effect is reversed, for the opacity arises mainly from the metals, and becomes very low when these are absent, so that a pure hydrogen star would again be bright. These principles, discussed by Eddington,* have been fully worked out by B. Strömgren, $\uparrow$ who finds that, if the coefficient in the opacity formula is taken strictly from theory, the observed luminosities usually lie about midway between the upper and lower limits, and can be accounted for by a percentage of hydrogen (by weight) which is close to 30 for most of the stars for which good data are available. (There is an alternative solution, with more than 99 per cent of hydrogen, which appears to be improbable.)

Strömgren, from rather weak data regarding certain eclipsing variables, concluded that the percentage of hydrogen in giant stars is considerably smaller; but this conclusion is not confirmed by your lecturer's just-completed work on visual double stars, and the question must remain sub judice for the present.

We are now faced with a serious problem. Why should the observed correlation between mass and luminosity be as close as it is? Differences in the hydrogen content alone may account for a range of over five magnitudes in the luminosity of a star of given mass. The extreme observed range (corrected for errors of observation) is perhaps one-third as great. The stars, in general, appear to be very similar in composition, or at least in

* Monthly Notices of the Royal Astronomical Society, vol. 92 (1932), p. 471.

$\dagger$ Zeitschrift für Astrophysik, vol. 4 (1932), p. 118. 
hydrogen content. The future explanation of this must be sought from nuclear physics-evidently no theory of stellar models can account for it.

It is noteworthy, however, that the observational evidence of similar hydrogen content comes entirely from main-sequence stars and giants, for which Vogt's Theorem indicated similarity of composition, at least within each group. We have as yet no data regarding the masses of super-giants, except for rather doubtful estimates for Cepheid variables.

Another apparent difficulty, however, is more easily solved. With the accepted expression for the opacity, the mass-luminosity relation takes the form

$$
L=\text { const. } M^{5.5} \beta^{7.5} \mu^{8.5} R^{-0.5} .
$$

On account of the factor $R^{-1 / 2}$, a star of given mass may be provided with any assigned luminosity by adjusting its radius. Why then do we find a mass-luminosity correlation? We may introduce the effective surface temperature $T_{e}$ in place of $R$, by the relation $L=$ const. $R^{2} T_{e}^{4}$, and so find

$$
L=\text { const. } M^{4.4} \beta^{6} \mu^{6.8} T_{e}^{0.8} .
$$

Now $L$ is the bolometric luminosity, corresponding to the total heat-radiation. For values of $T_{e}$ between $4000^{\circ}$ and $10,000^{\circ}$, a considerable percentage of this is in the spectral region accessible to visual or photographic observation. A five-fold increase or decrease of temperature would throw all but a beggarly fraction of the radiation into unobservable regions, so that observational selection would give us an apparent mass-luminosity correlation if no real one existed.

The remarkable correlations which exist between $L$ and $T_{e}$ for the actual stars make this explanation irrelevant. Their origin is again to be sought in nuclear physics.

An important addition to our stock of stellar models results from Milne's extensive work. He approaches the problem from a new direction. Previous investigators had assumed a knowledge of most, if not all, of the properties of stellar mattermolecular weight, opacity, energy-generation-and deduced the luminosity and radii of stars as functions of their masses. Milne starts with the information that a star has a given mass, 
luminosity, and radius, and inquires what configurations may possess these values. The results of the different lines of approach are well illustrated by the over-simplified but tractable standard model, in which $\epsilon$ and $k$ are supposed to be constant.

Eddington's original analysis showed that, in this case, a model of mass $M$, composed of perfect gas with given values of $k$ and $\mu$, must have exactly a certain luminosity $L_{0}$, though it may have any radius whatever. This solution enriched astrophysics with the mass-luminosity law; but it has what Jeans has quite justly called "weird and wonderful properties," for it demands that $\epsilon$ shall have the precise value $L_{0} / M$ and no other, that is, that a star of given mass must generate energy at exactly this rate if it is to be capable of permanent existence. These strange properties are, however, purely fictitious-a result of the arbitrary assumptions $k=$ const., $\mu=$ const. Under these conditions, the free parameter determining the set of solutions consistent with Vogt's Theorem is the radius $R$, and the weirdness of the results represents only the fact that the graph of such a set, in the $M R$ plane, is a horizontal straight line. If either $k$ or $\mu$ is supposed to vary with the physical conditions, the paradox vanishes (except for specialized functional forms such as $k=\rho / T^{3}$, which bring it back).

Milne, studying the configuration of a mass $M$, with the same constant $\mu$ and $k$, but a different $\epsilon$ (or $L$ ), was led to the solutions of Emden's equation which had previously been passed over because of the existence of central singularities.

If $L<L_{0}$, the solution, carried inward from the known values at the surface, exhausts the mass before the center is reached. A mass with a hole in the middle is physically absurd; but it is not absurd to ask what would become of a mass $M$, composed of real gas possessing the given properties. Milne shows that it would collapse and increase in density until the simple gaslaws failed to apply. The decreasing compressibility then makes a solution possible, with a high but finite central density, and a small radius. For $L>L_{0}$ the mass is not exhausted as $r$ tends to zero. If $L-L_{0}$ is small, equilibrium may be attained, formally, by placing a point-mass at the center, or, in a physically significant manner, by assuming the existence of a small core of high density, within which the equation of state is different. When, however, $L$ exceeds a certain limit $L_{1}$, equilibrium is impossi- 
ble, for the radiation pressure exceeds the gravitational, and the mass must expand to infinity.

Milne's later studies,* on a generalized standard model, in which $\epsilon$ is the same throughout, but $k$ is substantially zero in the degenerate core and constant in the envelope, show that the configurations of models of fixed mass, but different $\epsilon$ and $L$, will be of the collapsed type if $L<L_{0}$ (the value corresponding to Eddington's simple solution), and centrally condensed if $L>L_{0}$. Above a certain limit $L_{\max }$, no equilibrium configurations exist. Between this and $L_{0}$, there are two centrally-condensed solutions, differing in radius, size of core, and other properties. If all these configurations are arranged in order of the relative radius of the core, the series becomes linear.

Starting with the familiar diffuse solution, with no core, and $L=L_{0}$, the configurations with a small core are centrally condensed, with increasing $L$. For a certain core-radius $L$ is a maximum. So long as $L>L_{0}$, the configurations are still centrally condensed. The transition to the collapsed type takes place through a quasi-diffuse configuration of small radius, and with a degenerate core, but with the outer portion exactly the same as a diffuse configuration of the same size. Finally $L=0$ when the whole mass is degenerate and cold.

This linear series is not of the type predicted by Vogt's Theorem, for $\epsilon$, and hence the composition, varies along it. But, from a complete set of solutions of Milne's problem, for different values of $M$ ( $k$ and $\mu$ being the same throughout) it would be possible to pick these for which $L / M$ had a constant value $\epsilon_{0}$, and so obtain a series consistent with the theorem (since the transition from the perfect gas to the degenerate equation of state, though supposed for convenience to be abrupt, involves no disposable constants). From Milne's calculations it appears probable that the original diffuse configurations would have the greatest radius.

The collapsed models toward the end of this series, with large degenerate cores and small luminosities, afford an admirable representation of the white dwarfs, and a notable triumph for Milne's analysis. The earlier stages have not yet been definitely

* Monthly Notices of the Royal Astronomical Society, vol. 92 (1932), p. 610. 
matched with actual stars, but may be represented by the fainter red dwarfs.

However accurate our knowledge of the law of opacity, and the luminosity of individual stars might become, there is still great difficulty in the approach from this side, for the molecular weight in the sub-surface layers depends greatly on the abundance of hydrogen, and $L_{0}$ depends on this. Unless some way can be found for determining the hydrogen abundance $a$ priori-and accurately-it remains as a disposable parameter. By adjusting it, $L_{0}$ can be made equal to the observed $L$, over a wide range of the latter, and even the type of the configuration changed. Change of the radius with the size of the core affords a better hope of solution; but, unfortunately, the effective temperature, surface brightness, and diameter of a faint red star are very difficult to determine accurately by observation; so the question remains unsettled.

It is generally agreed that collapsed configurations are always of small radius, and so can represent only white dwarfs. Whether centrally condensed configurations can exist with large radii is still a matter of debate. Milne, from certain general considerations, believes it probable that they do (in advance of detailed calculations to settle the question). Eddington* and Chandrasekhar, $\dagger$ from a discussion of the outer region, or envelope, of a model star, conclude that they do not, but their latest work involves simplifying approximations.

There is a possibility of answering this question in quite a different way. In a close binary pair, the individual stars must be flattened at the poles by their rotation. If the orbit is eccentric, the periastron will then advance, at a rate depending not only on the masses and radii of the components (which are known from observation) but on the degree of central condensation. Several cases of apsidal motion of this sort are known, and in one, Y Cygni, a complete revolution of the major axis has been observed.

This hope of obtaining definite information about the internal density has been shattered by recent investigations of Walter. In the case which occurs most of ten, the rotation and revolution 109.

* Monthly Notices of the Royal Astronomical Society, vol. 91 (1931), p.

† Ibid., vol. 96 (1936), p. 647. 
periods of the stars coincide, and they are drawn out into ellipsoids by tidal forces. Treating these ellipsoids as rigid, Walter has shown that the motion becomes much more complicated. The major axes of the ellipsoids oscillate about the line joining their centers; there are then three free periods instead of one, and it is found that the line of apsides should slowly retrograde.

If the stars have broken loose from tidal control, and are spheroids, with rotation periods shorter than the orbital, the periastron advances - and it is noteworthy that, in all cases in which an advance has been observed, the components are rather small compared with their mutual distance, so that this hypothesis is reasonable. But, in this case, the exact rotation periods are unknown, and the resulting oblateness, and theoretical motions of the apse, share the uncertainty.

An extensive literature, and some controversy, has grown out of Milne's work. The difficulty has arisen largely from the great difference in the manner of approach to the question. Speaking roughly, Eddington and his school state it as a physical problem-what will happen to a mass of real matter, or, at least, of matter idealized no more than is necessary, to get something that can be handled analytically? Milne and his followers state a mathematical problem - given the mass, luminosity and radius of a body, what may be inferred regarding its internal constitution, assuming the exact validity of certain equations of state, and the like? The student whose thought proceeds habitually along either line has sometimes hard work not to misunderstand the other. One may sympathize with Eddington when he said years ago, in answer to doubts regarding the convergence of a series: "Surely this verifies my statement that $C$ is negligibly small. I need scarcely say that $\mathrm{I}$ have not suggested that we should neglect $10,000,000,000$ C." ; and equally with Milne, when he found that his expression, "a star endowed with sources of energy totalling to $L$," had been interpreted by his critics as "provided with sources of fixed total strength $L$," when he actually meant "possessing sources which, under the actual internal conditions, total $L "$-without hypotheses as to what they might be under other conditions. Both lines of approach are valid, and both have led to notable advances in astrophysical knowledge. When the underlying equa- 
tions rest upon well-established physical principles, they naturally lead to the same results-when misinterpretations and occasional casual slips have been cleared up. When no accepted physical basis is yet available, the postulates preferred by various investigators may differ, and the results diverge, but it is just in this way that knowledge is ultimately advanced, when some decisive test has been found.

One final phase of the subject demands discussion-which must now be brief. We have so far dealt only with the existence of equilibrium, and not at all with its stability. A stellar model may fail of permanence in several ways.

It may be in unstable gravitational equilibrium. The best example is a mass for which a small change in the total kinetic energy of the molecules and photons involves a change in the internal energy (of ionization, for example) of the same sign and greater in amount-in other words, one in which the ratio $\gamma$ of the specific heats is less than 4/3. A contraction of such a body would liberate a certain amount of gravitational energy, but call for the imparting of a greater amount to the mass to maintain it in equilibrium. It would therefore be out of balance, contract still further, and make things worse. A small expansion would release more energy than was required to lift the mass against gravity, and result in expansion at an increasing rate. A mass of perfect gas, with $\gamma<4 / 3$ (if such were possible), could therefore never be in stable equilibrium; it would either contract indefinitely or expand till it was dissipated altogether into space.

No such startling behavior need be feared for a star as a whole; but it is possible that in certain zones-where, for example, several electrons are being removed in rapid succession from the $L$-shells of abundant atoms-the effective value of $\gamma$ might fall locally below the safe limit. If such a state should be gradually approached-say during the slow contraction of the star-a rapid and catastrophic contraction of the affected zone might set in, resulting in a change of model, and a great liberation of gravitational energy, which, at the start, would appear as heat-sensible and latent-in the collapsed zone. Following an idea of Milne's, it may be surmised that an internal collapse of this sort (not very deep within the star) may supply the energy for the explosive outburst of a Nova. 
A star is in thermal equilibrium if it contains internal sources of heat sufficient to supply the loss by radiation. A disturbance, for example, by a homologous contraction, will alter the rate of loss-increasing it if $k \propto \rho T^{-7 / 2}$ and $L \propto R^{-1 / 2}$. If the sourcestrength is increased more than this, the star is stable. This happens because a star, as a whole, has a negative specific heat; contraction increases the internal temperature, but diminishes the total internal energy, when gravitation is taken into account. The generation of excess heat within it, after contraction, therefore causes expansion and restores the status quo.

A star with constant $\epsilon$ (for example, one deriving its energy from radio-activity) would therefore be thermally unstable. The rate of liberation of heat from sub-atomic sources, however, would presumably increase very rapidly with the temperature, and avoid this. The danger, in any case, would not be immediate, for, with the definition given here, a star which derived its energy wholly from gravitational contraction would be quite unstable; yet, if comparable with the sun, it would continue shining for millions of years.

The third danger is of pulsatory instability-or "overstability" as Eddington has called it. Under dynamical forces alone, a gravitating mass of gas, if compressed to less than its equilibrium volume, would expand, over-shoot the mark, and be left in oscillation - the fundamental type being a simple radial pulsation, or change of volume of a spherical mass. In an actual mass the leakage of heat from hotter to colder regions would tend to damp the oscillation out. The pulsation would alter the rate of generation of heat inside, and of escape from the surface; but a change in the mean radius would equalize the integrated values of the two over the period. If, however, the rate of generation increased rapidly with temperature, most of the heat would be liberated when the star was smallest, and thus provide a series of expansive impulses, timed just so as to increase the oscillation. If this effect was greater than the damping, the amplitude of pulsation would increase exponentially (at least until other modifying forces began to operate) and the star would be overstable.

Investigations by Eddington and Jeans indicate that this is to be feared, provided that $\epsilon$ increases much faster than $T^{2}$. This presents a very serious difficulty, and has led Sterne and others to believe that the source of energy is to be sought in small, 
highly condensed central regions, where, at a temperature exceeding $10^{9}$ degrees, reversible transformations of matter into radiation may occur. The difficulty has however been substantially removed by Cowling* who finds that, if $\epsilon$ increases rapidly with $T$, the temperature gradient will be so high that a zone of convection will be formed in the interior of a star. This leads to important changes in the density model, and, in particular, removes the holes of low central density which appear in some models carefully calculated from plausible laws of energy generation.

Cowling's analysis shows that $\epsilon$ may vary as rapidly as $T^{20}$ without causing over-stability, unless the value of $\gamma$ for the gas itself is close to $4 / 3$.

From one standpoint, the business of building model stars may be regarded as highly successful. Starting with the most general principles of physics, models have been constructed which closely represent the properties of stars-not of one sort alone, but of practically all types - and satisfy the conditions of stability. The mass-luminosity relation, and the enigmatical white dwarfs, have been explained.

From another aspect, most of the work is still to do. The analytical problems which remain - such as those presented by zones of convection - can probably be solved, approximately at least, by ingenuity in discussion and assiduity in quadratures. But the physical problems still baffle us. From the nature of the situation, no unique solution for the law of energy generation can be derived from a study of the properties of stars as a whole, even if these were known with high precision. There is a much better chance of being able to deduce the generation law from nuclear physics; but present knowledge indicates that this is likely to depend on the composition of the material in a complicated fashion, so that the problems of steady states and possible evolution of the stars, and of the relative proportions and possible transmutation of the elements within them are likely to be intimately connected, as Atkinson's pioneer work has shown.

We may not live to see the final success; but, when it comes, it will the more enlarge our knowledge of the Universe.

Princeton University

* Monthly Notices of the Royal Astronomical Society, vol. 96 (1935), p. 42. 